\title{
Estimates of the optimal density and kissing number of sphere packings in high dimensions
}

\author{
A. Scardicchid* \\ Department of Physics, Joseph Henry Laboratories, \\ Princeton University, Princeton NJ 08544 and \\ Princeton Center for Theoretical Physics, \\ Princeton University, Princeton NJ 08544 \\ F. H. Stillingent \\ Department of Chemistry, Princeton University, Princeton NJ 08544 \\ S. Torquatd \\ Department of Chemistry, Princeton University, Princeton NJ 08544 \\ Program in Applied and Computational Mathematics, \\ Princeton University, Princeton NJ 08544 \\ PRISM, Princeton University, Princeton NJ 08544 and \\ Princeton Center for Theoretical Physics, \\ Princeton University, Princeton NJ 08544
}




\begin{abstract}
The problem of finding the asymptotic behavior of the maximal density $\phi_{\max }$ of sphere packings in high Euclidean dimensions is one of the most fascinating and challenging problems in discrete geometry. One century ago, Minkowski obtained a rigorous lower bound on $\phi_{\max }$ that is controlled asymptotically by $1 / 2^{d}$, where $d$ is the Euclidean space dimension. An indication of the difficulty of the problem can be garnered from the fact that exponential improvement of Minkowski's bound has proved to be elusive, even though existing upper bounds suggest that such improvement should be possible. Using a statistical-mechanical procedure to optimize the density associated with a "test" pair correlation function and a conjecture concerning the existence of disordered sphere packings [S. Torquato and F. H. Stillinger, Experimental Math. 15, 307 (2006)], the putative exponential improvement on $\phi_{\max }$ was found with an asymptotic behavior controlled by $1 / 2^{(0.77865 \ldots) d}$. Using the same methods, we investigate whether this exponential improvement can be further improved by exploring other test pair correlation functions correponding to disordered packings. We demonstrate that there are simpler test functions that lead to the same asymptotic result. More importantly, we show that there is a wide class of test functions that lead to precisely the same putative exponential improvement and therefore the asymptotic form $1 / 2^{(0.77865 \ldots) d}$ is much more general than previously surmised. This class of test functions leads to an optimized average kissing number that is controlled by the same asymptotic behavior as the one found in the aforementioned paper.
\end{abstract}

PACS numbers: 05.20.-y, 61.20.-p

*Electronic address: ascardic@princeton.edu

${ }^{\dagger}$ Electronic address: fhs@princeon.edu

${ }^{\ddagger}$ Electronic address: torquato@electron.princeton.edu 


\section{INTRODUCTION}

A collection of congruent spheres in $d$-dimensional Euclidean space $\mathbb{R}^{d}$ is called a sphere packing if no two spheres overlap. Although the practical relevance of sphere packings in high Euclidean dimensions was appreciated by Shannon in 1948 [1], there has been a resurgence of interest in such problems in both the physical and mathematical sciences $[2,3,44,5,6,17,8,9,10,11]$. Shannon showed that the optimal way of sending digital signals over noisy channels corresponds to the densest sphere packing in a high dimensional space [1]. These "error-correcting" codes underlie a variety of systems in digital communications and storage [12]. Physicists have investigated sphere packings in high dimensions to gain insight into classical ground and glassy states of matter as well as phase behavior in lower dimensions [8, 9, 15, 16, 17]. Understanding the symmetries and other mathematical properties of the densest packings in arbitrary dimension is a problem of long-standing interest in discrete geometry [4, 5, 12, 13, 14].

The packing density or simply density $\phi$ of a sphere packing is the fraction of space $\mathbb{R}^{d}$ covered by the spheres. We will call

$$
\phi_{\max }=\sup _{P \subset \mathbb{R}^{d}} \phi(P)
$$

the maximal density, where the supremum is taken over all packings in $\mathbb{R}^{d}$. The set of lattice packings is a subset of the set of sphere packings in $\mathbb{R}^{d}$. A lattice $\Lambda$ in $\mathbb{R}^{d}$ is a subgroup consisting of the integer linear combinations of vectors that constitute a basis for $\mathbb{R}^{d}$. A lattice packing $P_{L}$ is one in which the centers of nonoverlapping spheres are located at the points of $\Lambda$. In a lattice packing, the space $\mathbb{R}^{d}$ can be geometrically divided into identical regions $F$ called fundamental cells, each of which contains the center of just one sphere. In the physical sciences, a lattice packing is simply a packings arranged on the sites of a Bravais lattice. Non-lattice packings include periodic packings (more than one sphere per fundamental cell) as well as disordered packings [18].

The sphere packing problem seeks to answer the following question: Among all packings of congruent spheres, what is the maximal packing density $\phi_{\max }$, i.e., largest fraction of $\mathbb{R}^{d}$ covered by the spheres, and what are the corresponding arrangements of the spheres [12, 14]? For arbitrary $d$, the sphere packing problem is notoriously difficult to solve. In the case of packings of congruent $d$-dimensional spheres, the exact solution is known for the first three 
space dimensions. For $d=1$, the answer is trivial because the spheres tile the space so that $\phi_{\max }=1$. In two dimensions, the optimal solution is the triangular lattice arrangement (also called the hexagonal packing) with $\phi_{\max }=\pi / \sqrt{12}$. In three dimensions, the Kepler conjecture that the face-centered cubic lattice arrangement provides the densest packing with $\phi_{\max }=\pi / \sqrt{18}$ was only recently proved by Hales [5]. For $3<d<10$, the densest known packings of congruent spheres are lattice packings (defined below). For example, the "checkerboard" lattice $D_{d}$, which is the $d$-dimensional generalization of the FCC lattice, is believed to be optimal in $\mathbb{R}^{4}$ and $\mathbb{R}^{5}$. The $E_{8}$ and Leech lattices in $\mathbb{R}^{8}$ and $\mathbb{R}^{24}$, respectively, are remarkable dense and symmetric and are most likely the densest packings in these dimensions [11]. However, for sufficiently large $d$, lattice packings are most likely not the densest, but it becomes increasingly difficult to find specific dense packing constructions in high dimensions [19]. In high dimensions, the best that one can do theoretically is to devise rigorous upper and lower bounds on $\phi_{\max }$.

Upper and lower bounds on the maximal density $\phi_{\max }$ exist in all dimensions [12]. Minkowski [20] proved that the maximal density $\phi_{\max }^{L}$ among all Bravais lattice packings for $d \geq 2$ satisfies the lower bound

$$
\phi_{\max }^{L} \geq \frac{\zeta(d)}{2^{d-1}},
$$

where $\zeta(d)=\sum_{k=1}^{\infty} k^{-d}$ is the Riemann zeta function. One observes that for large values of $d$, the asymptotic behavior of the nonconstructive Minkowski lower bound is controlled by $2^{-d}$. Since 1905, many extensions and generalizations of (2) have been obtained [12], but none of these investigations have been able to improve upon the dominant exponential term $2^{-d}$. The best currently known rigorous lower bound on $\phi_{\max }^{L}$ was obtained by Ball [21]. He found that

$$
\phi_{\max }^{L} \geq \frac{2(d-1) \zeta(d)}{2^{d}} .
$$

Interestingly, the density of a saturated packing of congruent spheres in $\mathbb{R}^{d}$ for all $d$ satisfies the lower bound [22]

$$
\phi \geq \frac{1}{2^{d}}
$$

and thus has the same dominant exponential term as the Minkowski lower bound (2). A saturated packing of congruent spheres of unit diameter and density $\phi$ in $\mathbb{R}^{d}$ has the property that each point in space lies within a unit distance from the center of some sphere. As we will discuss below, the lower bound (4) is not a stringent bound for a saturated packing and 
therefore is improvable.

Rogers [13, 14] found upper bounds on the maximal density $\phi_{\max }$ by an analysis of the Voronoi cells. For large $d$, Rogers' upper bound asymptotically becomes $d 2^{-d / 2} / e$. Kabatiansky and Levenshtein [23] found an even stronger bound, which in the limit $d \rightarrow \infty$ yields $\phi_{\max } \leq 2^{-0.5990 d(1+o(1))}$. Cohn and Elkies [4] obtained and computed linear programming upper bounds, which provided improvement over Rogers' upper bound for dimensions 4 through 36. They also conjectured that their approach could be used to prove sharp bounds in 8 and 24 dimensions. Indeed, Cohn and Kumar [11] used these techniques to prove that the Leech lattice is the unique densest lattice in $\mathbb{R}^{24}$. They also proved that no sphere packing in $\mathbb{R}^{24}$ can exceed the density of the Leech lattice by a factor of more than $1+1.65 \times 10^{-30}$, and gave a new proof that the $E_{8}$ lattice is the unique densest lattice in $\mathbb{R}^{8}$.

A recent investigation [6] proves that there exists a disordered packing construction in $\mathbb{R}^{d}$ with a maximal density that achieves the saturation lower bound (4) for any $d$. This construction is referred to as the "ghost" random sequential addition (RSA) packing [24] and it was shown that all of the $n$-particle correlation functions for this packing can be obtained analytically for all allowable densities and in any dimension. Interestingly, this packing is unsaturated (see Fig. 1) and yet it has a maximal density $2^{-d}$, which suggests that there exist disordered saturated packings that exceeds the saturation lower bound (4) or the Minkowski lower bound (2). Indeed, another recent study [10] strongly suggests that the standard disordered RSA packing [25] at its maximal saturation density scales as $d 2^{-d}$ for large $d$, which has the same asymptotic behavior as Ball's lower bound (3). Note that spheres in both the ghost and standard RSA packings cannot form interparticle contacts, which appears to be a crucial attribute to obtain exponential improvement on Minkowski's bound [7], as we discuss below.

Do there exist disordered packings that can provide the long-sought exponential improvement of Minkowski's lower bound? Torquato and Stillinger [7] employed a conjecture concerning the existence of disordered sphere packings and an optimization procedure that maximizes the density associated with a "test" pair correlation function to provide the putative exponential improvement on Minkowski's 100-year-old bound on $\phi_{\max }$ (see Section II for details). The asymptotic behavior of the conjectural lower bound is controlled by $2^{-((0.77865 \ldots)) d}$. Moreover, this lower bound always lies below the density of the densest known 


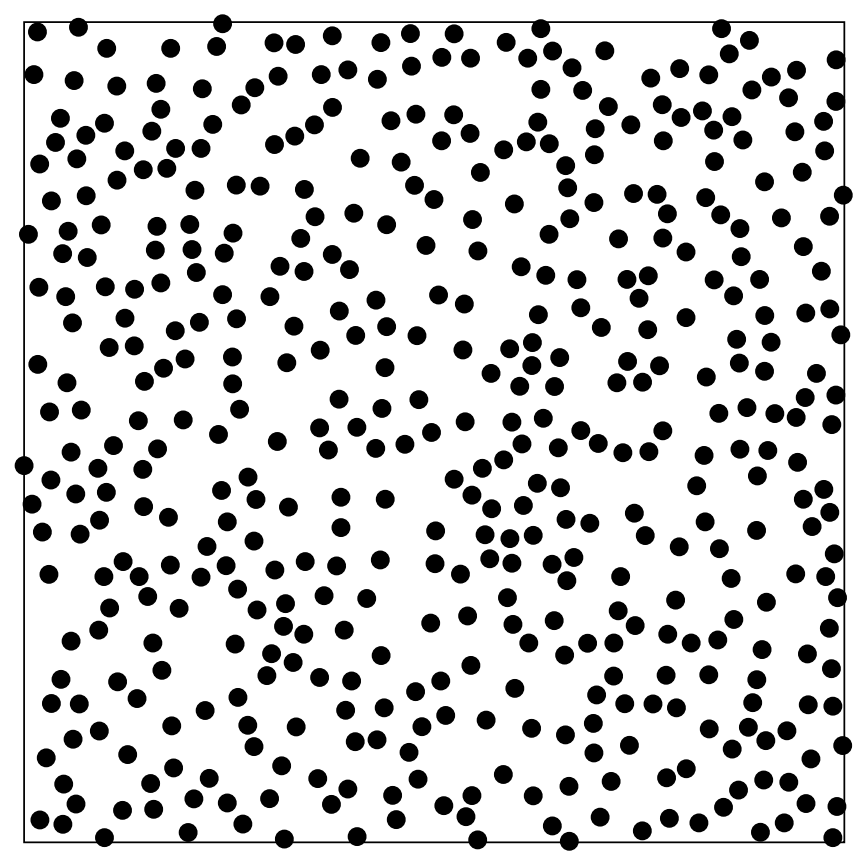

FIG. 1: (Color online) A configuration of 468 particles of a ghost RSA packing in $\mathbb{R}^{2}$ at a density very near its maximal density of 0.25 . This was generated using a Monte Carlo procedure within a square fundamental cell under periodic boundary conditions. Note that the packing is clearly unsaturated and there are no contacting particles.

packings for $3 \leq d \leq 56$, but, for $d>56$, it can be larger than the density of the densest known arrangements, all of which are ordered. These results counterintuitively suggest that the densest packings in sufficiently high dimensions may be disordered rather than periodic, implying the existence of disordered classical ground states for some continuous potentials. In addition, a decorrelation principle for disordered packings was identified in Ref. [7], which states that unconstrained correlations in disordered sphere packings vanish asymptotically in high dimensions and that the $g_{n}$ for any $n \geq 3$ can be inferred entirely (up to some small error) from a knowledge of the number density $\rho$ and the pair correlation function $g_{2}(\mathbf{r})$. This decorrelation principle [26], among other things, provides justification for the conjecture used in Ref. [7], and is vividly and explicitly exhibited by the exactly solvable ghost RSA packing process [6] as well as by computer simulations in high dimensions of the maximally random jammed state [9] and the standard RSA packing process [10].

In this paper, we investigate whether the putative exponential improvement of Minkowski's lower bound found in Ref. [7] can be further improved by exploring other 
test pair correlation functions. We will show that there are simpler test functions that lead to the same asymptotic result. More importantly, we will demonstrate that there is a wide class of test functions that lead to the same exponential improvement as in Ref. [7].

\section{PRELIMINARIES AND OPTIMIZATION PROCEDURE}

A packing of congruent spheres of unit diameter is simply a point process in which any pair of points cannot be closer than a unit distance from one another [7]. A particular configuration of a point process in $\mathbb{R}^{d}$ is described by the "microscopic" density

$$
n(\mathbf{r})=\sum_{i=1}^{\infty} \delta\left(\mathbf{r}-\mathbf{x}_{i}\right)
$$

This distribution can be interpreted in a probabilistic sense [7], which is particularly useful for the arguments we will present, even in the limit in which no explicit randomness is present, as in the case in which the spheres are arranged on the sites of a (Bravais) lattice. We define the $n$-particle density as the ensemble average

$$
\rho_{n}\left(\mathbf{r}_{1}, \ldots, \mathbf{r}_{n}\right)=\left\langle\sum_{i_{1} \neq i_{2} \neq \ldots \neq i_{n}} \delta\left(\mathbf{r}_{1}-\mathbf{x}_{i_{1}}\right) \ldots \delta\left(\mathbf{r}_{n}-\mathbf{x}_{i_{n}}\right)\right\rangle,
$$

which is a nonnegative quantity. Henceforth, we will assume that the random process is translationally invariant, i.e., statistically homogeneous. It follows that there is no preferred origin in the packing and thus the $n$-particle densities $\rho_{n}\left(\mathbf{r}_{12}, \mathbf{r}_{13}, \ldots, \mathbf{r}_{1 n}\right)$ only depend on relative displacements, where $\mathbf{r}_{1 j} \equiv \mathbf{r}_{j}-\mathbf{r}_{1}$. In particular, the one-particle density $\rho_{1}(\mathbf{r})=$ $\left\langle\delta\left(\mathbf{r}-\mathbf{x}_{1}\right)\right\rangle=\rho$ is a constant called the number (center) density. Note that the packing density $\phi$ defined earlier is related to the number density $\rho$ for spheres of unit diameter via the relation

$$
\phi=\rho v_{1}(1 / 2)
$$

where $v_{1}(r)=\pi^{d / 2} r^{d} / \Gamma(d / 2+1)$ is the volume of a sphere of radius $r$. The surface area of such a sphere is $s_{1}(r)=2 \pi^{d / 2} r^{d-1} / \Gamma(d / 2)$. If we divide $\rho_{n}$ by $\rho^{n}$, we get the $n$-particle correlation function $g_{n}\left(\mathbf{r}_{12}, \mathbf{r}_{13} \ldots, \mathbf{r}_{1 n}\right)$, which clearly is also a nonnegative function. As will become clear shortly, the pair correlation function $g_{2}\left(\mathbf{r}_{12}\right)$ has particular importance to us. If the point process is additionally rotationally invariant (i.e., the packing is statistically homogeneous and isotropic), the pair correlation function $g_{2}(r)$ depends only on the distance $r \equiv|\mathbf{r}|$. 
In Ref. [2], $g_{2}$-invariant processes were examined in order to gain insights about the nature of disordered sphere packings. A $g_{2}$-invariant process is one in which a given nonnegative pair correlation $g_{2}(\mathbf{r})$ function remains invariant for all $\mathbf{r}$ over the range of densities

$$
0 \leq \phi \leq \phi_{*}
$$

The terminal density $\phi_{*}$ is the maximum achievable density for the $g_{2}$-invariant process subject to satisfaction of certain necessary conditions on the pair correlation. In particular, they considered those "test" $g_{2}(r)$ 's that are distributions on $\mathbb{R}^{d}$ depending only on the radial distance $r$. For any test $g_{2}(r)$ associated with a packing, i.e., $g_{2}(r)=0$ for $r<1$, they maximized the corresponding density $\phi$, i.e.,

$$
\max \phi
$$

subject to the following two conditions:

$$
\begin{gathered}
g_{2}(r) \geq 0 \quad \text { for all } r, \\
S(k)=1+\rho(2 \pi)^{d / 2} \int_{0}^{\infty} d r r^{d-1} \frac{J_{d / 2-1}(k r)}{(k r)^{d / 2-1}}\left[g_{2}(r)-1\right] \geq 0 \quad \text { for all } k .
\end{gathered}
$$

Condition (11) states that the structure factor $S(k)$ [trivially related to the Fourier transform of $g_{2}(r)-1$ ] must also be nonnegative for all wavenumbers. It is a known necessary condition on the existence of a point process [2, 27], but it is generally not sufficient [28].

Recently, Torquato and Stillinger [7] conjectured that a disordered sphere packing in $\mathbb{R}^{d}$ at number density $\rho$ exists for sufficiently large $d$ if and only if the conditions (10) and (11) are satisfied. The maximum achievable density is the terminal density $\phi_{*}$, which then implies the lower bound

$$
\phi_{\max } \geq \phi_{*}
$$

There is mounting evidence to support this conjecture. First, the aforementioned decorrelation principle states that unconstrained correlations in disordered sphere packings vanish asymptotically in high dimensions and that the $g_{n}$ for any $n \geq 3$ can be inferred entirely from a knowledge of $\rho$ and $g_{2}$. Second, other necessary conditions on $g_{2}$, such as the Yamada condition [29] as well as others [7], appear to only have relevance in very low dimensions. Third, one can recover the form of known rigorous bounds [cf. (2) and (3)] for specific test $g_{2}$ 's when the conjecture is invoked. Finally, in these two instances, configurations of 
disordered sphere packings on the torus have been numerically constructed with such $g_{2}$ in low dimensions for densities up to the terminal density [30, 31].

Interestingly, the optimization problem defined above is the dual of the infinitedimensional linear program devised by Cohn and Elkies [3, 4] to obtain upper bounds on the maximal packing density. In particular, let $f(r)$ be a radial function in $\mathbb{R}^{d}$ such that

$$
\begin{aligned}
& f(r) \leq 0 \quad \text { for } \quad r \geq 1 \\
& \tilde{f}(k) \geq 0 \quad \text { for all } k .
\end{aligned}
$$

Then the number density $\rho$ is bounded from above by

$$
\min \frac{f(0)}{2^{d} \tilde{f}(0)}
$$

The radial function $f(r)$ can be physically interpreted to be a pair potential. The fact that its Fourier transform must be nonnegative for all $k$ is a well-known stability condition for many-particle systems with pairwise interactions [32]. We see that whereas the linear program specified by (9), (10) and (11) utilizes information about pair correlations, its dual program (13) and (14) employs information about pair interactions. It is important to note [7] that even if there does not exist a sphere packing with $g_{2}$ satisfying conditions (10) and (11), the terminal density $\phi_{*}$ can never exceed the Cohn-Elkies upper bound. Every linear program has a dual program and when an optimal solution exists, there is no duality gap between the upper bound and lower bound formulations. However, until recently, it was not clear how to prove that there was no duality gap for the aforementioned infinite-dimensional sphere-packing linear program [3]. Recently, Cohn and Kumar [33] have proved that there is no duality gap.

By means of the linear program described above and the aforementioned conjecture concerning the existence for a certain test function $g_{2}$, it was found in Ref. [7] that in the limit $d \rightarrow \infty$,

$$
\phi_{\max } \geq \phi_{*} \sim 2^{-\frac{3}{2} d+\frac{1}{\ln 2} \frac{d}{2}+2.12497 \ldots d^{1 / 3}+\frac{1}{6} \log _{2} d+\log _{2}(3.2761 \ldots)},
$$

where the terms neglected are monotonically decreasing with $d$. The first term in the series provides the putative exponential improvement of Minkowski's lower bound (2). In the following, we will be interested mainly in the exponential improvement of Minkowski's lower bound, and so we simplify the right-hand side of (15) by writing it as

$$
\phi_{*} \sim 2^{-\left(\frac{3}{2}-\frac{1}{2 \ln 2}\right) d}=2^{-0.77865 \ldots d} .
$$


This is not to be intended as an asymptotic expansion of $\phi_{*}$ in the sense of Poincaré (the ratio of the right-hand side to the left-hand side does not go to unity when $d \rightarrow \infty$ ), however, it is an asymptotic expansion in such sense for $\log _{2} \phi_{*}$.

In what follows, we will show that we can obtain a conjectural lower bound asymptotically equal to (15) with a simpler test function. Then we will demonstrate that the requirement

of hyperuniformity [27] in Ref. [7] is actually a necessary condition that arises only from the optimization procedure. Finally, we will show some examples of how enlarging the space of test functions where the optimization is performed does not change the asymptotic exponential behavior, although non-exponential improvement is found.

Although these results do not constitute a proof of lower bounds, they strongly suggest that an estimate of the asymptotic behavior of the solutions to the lower-bound linear programming problem can be achieved and that physical intuition is gained about the spatial structures they describe.

\section{STEP PLUS DELTA FUNCTION REVISITED}

Following Torquato and Stillinger [7], we choose the following test $g_{2}(r)$ :

$$
g_{2}(r)=\Theta(r-1)+\frac{Z}{s_{1}(1) \rho} \delta(r-1) .
$$

Here the parameter $Z$ has the interpretation of the average kissing number. The structure factor becomes

$$
\begin{aligned}
S(k) & =1-2^{d / 2} \Gamma\left(1+\frac{d}{2}\right) \frac{J_{d / 2}(k)}{k^{d / 2}} 2^{d} \phi+2^{d / 2-1} \Gamma\left(\frac{d}{2}\right) \frac{J_{d / 2-1}(k)}{k^{d / 2-1}} Z \\
& \equiv 1-a(k) 2^{d} \phi+b(k) Z
\end{aligned}
$$

which defines the functions $a, b$. The terminal density is defined by the linear program (9), (10) and (11). $Z$ is then a free parameter to be optimized appropriately.

Unlike Torquato and Stillinger [7], we do not impose hyperuniformity [27] (requiring the structure factor to vanish at $k=0$ ) to simplify the optimization. Moreover, we are also interested in finding the largest average kissing number $Z$ that (for a given $d$ ) satisfies the constraints. In this latter case, it is $\phi$ that must be chosen appropriately. These are two infinite-dimensional, linear programming problems.

There is a graphical construction that will help us look for such points and that will be helpful also in cases where more parameters are to be varied. For any given $k$ the set of 
allowed points in the $(\phi, Z)$ plane [i.e., those for which $S(k) \geq 0$ ] is the half plane above (below) the line $1-a(k) 2^{d} \phi+b(k) Z=0$ for positive (negative) $a$. Upon changing $\mathrm{k}$ by a small step to $k+\Delta$, we repeat the construction and find the intersection of the two halfplanes. By letting $k$ vary over the positive reals and letting $\Delta \rightarrow 0$, we find a limiting finite, convex region $\mathcal{B}$ which gives the allowed values of $\phi, Z$. This region is the set internal to the curve obtained by solving the equations

$$
S(k, \phi, Z)=0, \quad \frac{\partial}{\partial k} S(k, \phi, Z)=0
$$

with respect to $\phi, Z$. This is depicted in Fig, 2 . It is not difficult to prove that the region $\mathcal{B}$ is indeed internal to the entire spiral. It will suffice to observe that the distance of a point on the spiral from the origin is a monotonically increasing function (for sufficiently large $k$ ).

Now the terminal density $\phi_{*}$ is the $x$-component of the rightmost point in $\mathcal{B}$. Analogously the $y$-component of the topmost point in $\mathcal{B}$ gives the terminal kissing number $Z_{* *}$.

The terminal density is found at the first zero of $b(k)$, which is located at the first zero of the Bessel function of order $d / 2-1$. As customary we call this number $j_{d / 2-1,1}$. The value of $\left(\phi_{*}, Z_{*}\right)$ is then found by finding the point on the spiral corresponding to $k=j_{d / 2,1}$ :

$$
\begin{aligned}
\phi_{*} & =\frac{2^{-d}}{a\left(j_{d / 2-1,1}\right)}=2^{-3 d / 2} \frac{\left(j_{d / 2-1,1}\right)^{d / 2}}{\Gamma(1+d / 2) J_{d / 2}\left(j_{d / 2-1,1}\right)}, \\
Z_{*} & =\frac{a^{\prime}\left(j_{d / 2-1,1}\right)}{b^{\prime}\left(j_{d / 2-1,1}\right) a\left(j_{d / 2-1,1}\right)}=\frac{a^{\prime}\left(j_{d / 2-1,1}\right)}{b^{\prime}\left(j_{d / 2-1,1}\right)} 2^{d} \phi_{*} .
\end{aligned}
$$

By using the asymptotic formulas, valid for large $\nu$

$$
\begin{aligned}
j_{\nu, 1} & =\nu+1.85576 \ldots \nu^{1 / 3}+\mathcal{O}\left(\nu^{-1 / 3}\right), \\
J_{\nu}\left(j_{\nu-1,1}\right) & =-J_{\nu-1}^{\prime}\left(j_{\nu-1,1}\right)=1.11310 \ldots \nu^{-2 / 3}+\mathcal{O}\left(\nu^{-4 / 3}\right),
\end{aligned}
$$

we find

$$
\phi_{*} \simeq 2^{-\frac{3}{2} d+\frac{1}{\ln 2} \frac{d}{2}+2.12497 \ldots d^{1 / 3}} \sim 2^{-(0.77865 \ldots) d} .
$$

Notice that this is the same case that was treated in [7] but there hyperuniformity was imposed and the Minkowski bound was recovered. Here we are not imposing hyperuniformity and the resulting terminal structure factor is not hyperuniform. The form of $S(k)$ at the terminal point $\phi_{*}, Z_{*}$ is given in Figure 2. Notice that the first zero is at $k=j_{d / 2-1,1} \simeq d / 2$. This can be interpreted as the appearance of a structure with length-scale $\ell \sim 1 / d$ in the 

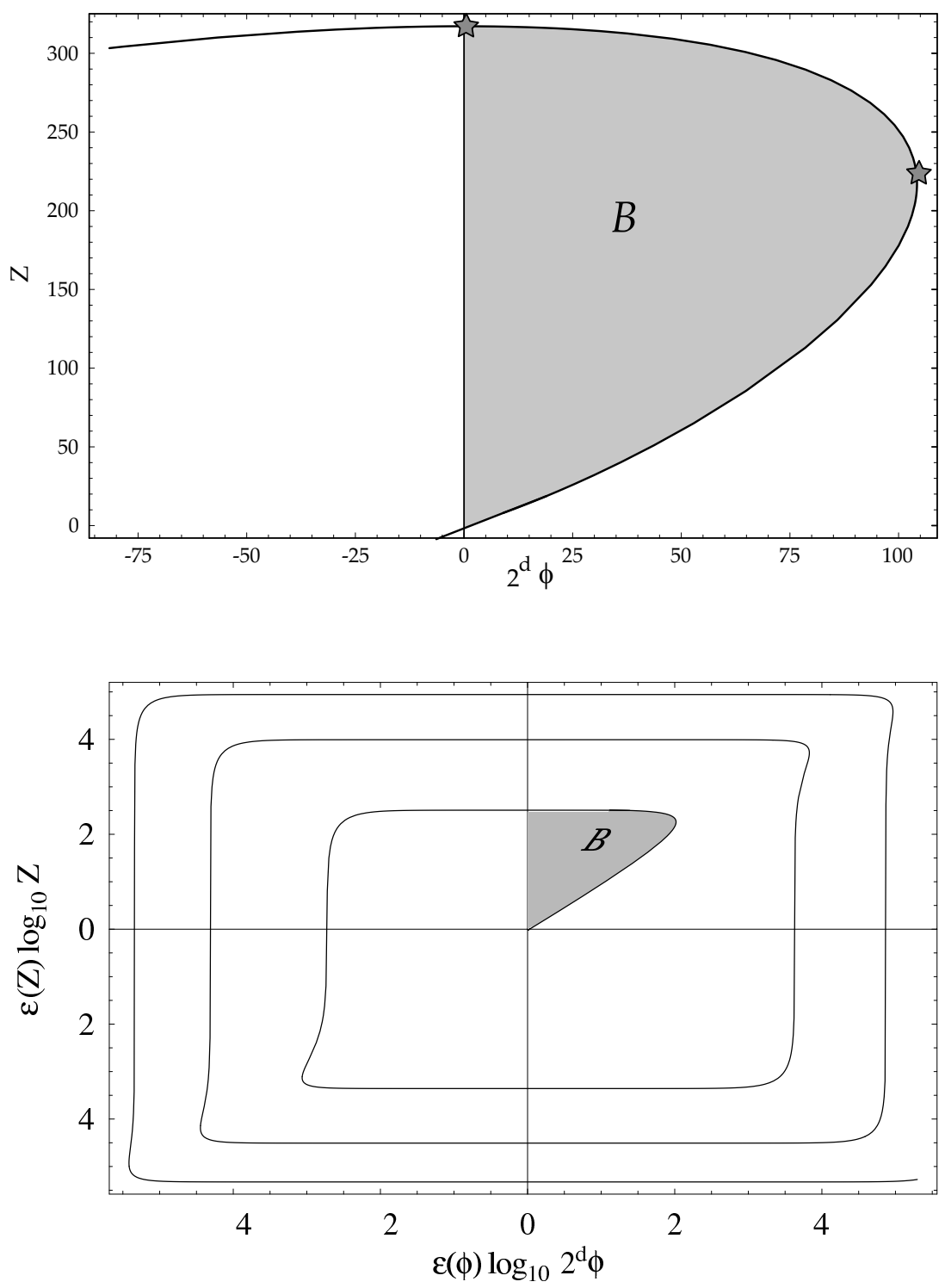

FIG. 2: (Top panel) For $d=16$, the set $\mathcal{B}$ of allowed packing densities and kissing numbers. The rightmost point is the maximal packing density $\phi_{*}$ and its corresponding kissing number $Z_{*}$. The topmost point is the maximal kissing number $Z_{* *}$ which corresponds to packing density $\phi_{* *}=0$. (Bottom panel) As in top panel, the region $\mathcal{B}$ of allowed packing densities and kissing numbers for $d=16$. For convenience in plotting, the horizontal and vertical axes represent the functions $\epsilon(\phi) \log _{10}\left(\left|2^{d} \phi\right|\right)$, and $\epsilon(Z) \log _{10}(|Z|)$, where $\epsilon(x)=\operatorname{sign} x$, respectively (although in this way the small region $\left|2^{d} \phi\right|<1,|Z|<1$ had to be left out of the graph). This figure shows how the solution of the equations $S(k, \phi, Z)=0, \partial S(k, \phi, Z) / \partial k=0$ for varying $k$ form an ever-growing spiral in which the allowed region $\mathcal{B}$ is completely contained. So this geometrical construction proves that every point in $\mathcal{B}$ are solutions to the linear programming problem $S(k, \phi, Z) \geq 0, \phi \geq 0, Z \geq 0$ for every $k \geq 0$. 


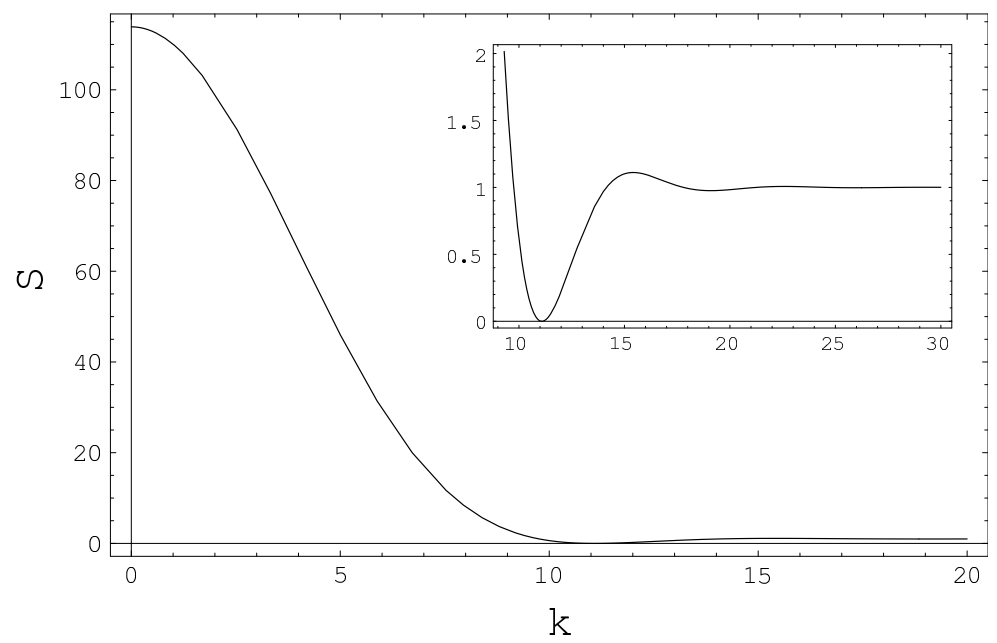

FIG. 3: The structure factor for the terminal density $\phi_{*}=0.0046692, Z_{*}=217.121$ in $d=16$. Notice the zero at $k^{*}=j_{7,1}=11.086 \ldots$ and the rapid asymptoting to the value $S(\infty)=1$.

system at large $d$. However, since a sphere packing corresponding to such an $S(k)$ could not be hyperuniform, it cannot be a Bravais lattice.

Following [7], we check whether the Yamada condition [29] on the number variance [29] is satisfied by the pair correlation (17). As in [7], we find a violation only for $d=1$.

The terminal kissing number is given by the topmost point in $\mathcal{B}$ which is the point $k^{* *}$ where $a\left(k^{* *}\right)=0$. It can be easily proved that $b^{\prime}\left(k^{* *}\right)=0$ as well so that $\phi_{* *}=0$ and

$$
Z_{* *}=-\frac{1}{b\left(j_{d / 2,1}\right)} \sim 2^{\left(\frac{1}{\ln 2}-1\right) \frac{d}{2}}
$$

It is intriguing to notice that the density corresponding to the terminal kissing number is zero.

\section{STEP PLUS DELTA FUNCTION WITH A GAP}

This case was analyzed by [7] before by imposing hyperuniformity. Here we show that in order to find the terminal density, one does not need to impose hyperuniformity from the beginning but rather that it arises as a necessary condition form the optimization procedure. We will show that the same asymptotic behavior of the terminal density found in the previous example is obtained (modulo non-exponential prefactors). 
We choose the test function

$$
g_{2}(r)=\Theta(r-(1+\sigma))+\frac{Z}{s_{1}(1) \rho} \delta(r-1),
$$

depending on two parameters, $Z, \sigma$ and the density of centers $\rho$. Performing the integrals gives the corresponding structure factor

$$
S(k)=1-a((1+\sigma) k) 2^{d}(1+\sigma)^{d} \phi+b(k) Z,
$$

where the functions $a, b$ were defined in the previous section. Again we look for the rightmost point in the set, which is now given by

$$
\begin{aligned}
& \phi_{*}=\frac{2^{-d}}{(1+\sigma)^{d} a\left((1+\sigma) j_{d / 2-1,1}\right)} \\
& Z_{*}=\frac{(1+\sigma) a^{\prime}((1+\sigma) k)}{b^{\prime}\left(j_{d / 2-1,1}\right) a\left((1+\sigma) j_{d / 2-1,1}\right)} .
\end{aligned}
$$

We now need to maximize the value of $\phi_{*}$ over $\sigma$. Clearly, we can increase $\sigma$ to increase $\phi_{*}$ indefinitely until $a\left((1+\sigma) j_{d / 2-1,1}\right)$ becomes zero, namely when $(1+\sigma) j_{d / 2-1,1}=j_{d / 2,1}$, which gives $\sigma \sim 2 / d$. The prefactor goes to a constant: $(1+\sigma)^{d} \sim(1+2 / d)^{d} \sim e^{2}$ and does not change the asymptotic dependence on $d$. This would suggest that the density can be increased without bound by adjusting the other parameters. This is not the case, however, since when we increase $\sigma$ we encounter the first "global" obstacle [by which we mean at wavenumbers $k$ far from the first zero of $b(k)$, which was setting the relevant $k$ scales up to now] at the value of $\sigma$ when $(1+\sigma)^{d} 2^{d} \phi_{*}=Z_{*}-1$. Notice that $a(0)=$ $b(0)=1$ and both functions decrease monotonically until their first zeros; here we have $S(0)=1-(1+\sigma)^{d} 2^{d} \phi_{*}+Z_{*}=0$ and any further increase of $\sigma$ would make $S(0)<0$. Thus, hyperuniformity has arisen as an optimality condition. Of course one should make sure that there is not a disconnected region in the parameter space $(\sigma, \phi, Z)$ with better terminal density $\phi_{*}$ but where hyperuniformity does not hold. We have searched the parameter space by discretizing the relevant range of $k$ and solving, using Mathematica, the linear program (9), (10) and (11). We have not been able to find another allowed region of the parameters disconnected from the previous one.

Hence we assume that the global terminal value $\phi_{*}$ is indeed obtained by imposing hyperuniformity and maximizing with respect to the remaining parameters (the two operations can be performed in any order). 
We notice that now we have reduced the problem to the case that has been analyzed in [7]. We will not repeat that analysis here but refer the reader to that paper. It is important to observe that in [7] the resultant asymptotic scaling law for the terminal fraction $\phi_{*}$

coincides with the one presented in the previous section $\phi_{*} \sim 2^{-(0.77865 \ldots) d}$. Although the non-exponential terms are different from those in the previous section, it is remarkable that the same exponential scaling law arises for two different cases. This strongly suggests that a large class of test functions can possess this asymptotic behavior. With this in mind, we go on to analyze the next case win which the test pair correlation function consists of a hard core with two delta functions and a gap.

\section{STEP PLUS TWO DELTA FUNCTIONS WITH A GAP}

In this Section we find the solution of the optimization problem (9), (10) and (11) for the family of pair correlation functions $g_{2}(r)$ composed of unit step function plus a gap and two delta functions, one at contact and the other at the end of the gap:

$$
g_{2}(r)=\theta(r-(1+\sigma))+\frac{Z_{2}}{s(1) \rho} \delta(r-1)+\frac{Z_{1}}{s(1+\sigma) \rho} \delta(r-(1+\sigma)) .
$$

This family depends on 3 parameters, $\sigma, Z_{1}, Z_{2}$ and we need to optimize them in order to find the optimal terminal density $\phi_{*}$. The structure factor is

$$
\begin{aligned}
S(k) & =1+Z_{2} 2^{d / 2-1} \Gamma(d / 2) \frac{J_{d / 2-1}(k)}{k^{d / 2-1}}+Z_{1} 2^{d / 2-1} \Gamma(d / 2) \frac{J_{d / 2-1}(k(1+\sigma))}{(k(1+\sigma))^{d / 2-1}}+ \\
& -\phi \Gamma(d / 2+1)(1+\sigma)^{d} 2^{3 d / 2} \frac{J_{d / 2}((1+\sigma) k)}{(k(1+\sigma))^{d / 2}} \\
& \equiv 1+Z_{2} c(k)+Z_{1} b(k)-(1+\sigma)^{d} 2^{d} \phi a(k),
\end{aligned}
$$

where the last line defines the functions $a, b, c$. Notice that $a(0)=b(0)=c(0)=1$ and $|a(k)|,|b(k)|,|c(k)| \leq 1$ follow from the properties of the Bessel functions. It is also convenient to reabsorb the factor $(1+\sigma)^{d} 2^{d}$ in the definition of $\phi$, i.e. $(1+\sigma)^{d} 2^{d} \phi \rightarrow \phi$. We will restore the proper units at the end of the calculation. The solution of this optimization problem for arbitrary $d$ is a formidable task. However, guided by the results of the previous section, we assume we can find an improvement on the previous bound even after imposing hyperuniformity.

Therefore, we fix the value of $Z_{2}=\phi-Z_{1}-1$ and are left with the other two parameters 
to optimize. Inserting this value of $Z_{2}$ in (32), we find the reduced optimization problem

$$
S(k)=(1-c(k))-(a(k)-c(k)) \phi+(b(k)-c(k)) Z_{1} \geq 0 .
$$

By using the fact that $c(k) \leq 1$ we might as well study the optimization problem

$$
\begin{aligned}
S^{(1)}\left(k, \sigma, \phi, Z_{1}\right) & \equiv \frac{S(k)}{1-c(k)} \equiv 1-\alpha(k) \phi+\beta(k) Z_{1} \geq 0 \\
\alpha(k) & =\frac{a(k)-c(k)}{1-c(k)}, \\
\beta(k) & =\frac{b(k)-c(k)}{1-c(k)} .
\end{aligned}
$$

Formally, this problem is analogous to the previous case with one delta function with gap and can be studied in the very same fashion. The process of having solved for $Z_{2}$ and changed the functions $a, b$ to $\alpha, \beta$ can be thought of as a renormalization process that allows to integrate out one delta function to reduce the problem to a simpler one.

The mathematical problem of finding the terminal fraction is formally identical to that of the previous section, although the constitutive functions $\alpha, \beta$ are more complicated. However, as long as a numerical analysis is concerned this does not present further difficulties.

We proceed in the following way: for a fixed $\sigma$ we find the rightmost point of allowed region, $\phi_{*}(\sigma), Z_{1, *}(\sigma)$, by finding the first zero of $\beta(k)$, call it $k^{*}$,

$$
\begin{aligned}
\phi_{*}(\sigma) & =\frac{1}{\alpha\left(k^{*}\right)}, \\
Z_{1, *}(\sigma) & =\frac{\alpha^{\prime}\left(k^{*}\right)}{\beta^{\prime}\left(k^{*}\right) \alpha\left(k^{*}\right)} .
\end{aligned}
$$

We then maximize the value of $\phi_{*}(\sigma)$ with respect to variations of $\sigma$. Generically, increasing $\sigma$ increases the value of $\phi_{*}$ until a positivity condition is violated (for small $k$ ). It turns out that the first condition to be violated is $S^{(1)}(0) \geq 0$. So in practice we find the terminal value of $\sigma$ by solving the equation

$$
S^{(1)}\left(0, \sigma, \phi_{*}(\sigma), Z_{1, *}(\sigma)\right)=0
$$

with respect to $\sigma$. Notice that this is now a "strong" hyperuniformity requirement, since $S^{(1)}(k) \sim k^{2}$ near the origin implies $S(k) \sim k^{4}$ near the origin, since $1-c(k) \sim k^{2}$. We are tempted to conjecture that this is a universal feature: adding more delta-functions to $g_{2}$ and solving the linear programming problem, we obtain structure factors $S(k)$ that become 
increasingly flatter at the origin. Hence, at least in this respect, the structure factor looks increasingly similar to that of a lattice.

As can be seen from Table 1 and Figure 4 (here the proper normalization for $\phi$ has been restored) the improvement on the previous bound is relevant but the asymptotic exponent is the same. Analytically, it is not difficult to obtain the rate of exponential decay (dictated mainly by the Stirling expansion of the gamma functions and the scaling of the first zero of $\beta$ with $d$ for large $d$ ), which turns out to be the same as the previous cases, namely,

$$
\phi_{*} \sim 2^{-(3 / 2-1 / 2 \ln 2) d}
$$

It is plausible, therefore, that the incorporation of any finite number of delta functions in a test $g_{2}$ will not improve the exponent in (40). This exponent fits the numerical data very well. A best fit of the data in Table II using the functions $d, d^{1 / 3}, \log _{2} d$, appearing in the analysis in the previous section and invoking the existence conjecture of Ref. [7] yields the putative lower bound

$$
\phi_{\max } \geq \phi_{*} \simeq 2^{-(0.77865 \ldots) d+2.12( \pm 0.04) d^{1 / 3}+0.39( \pm 0.08) \log _{2}(d)+\ldots} .
$$

The first term is fixed by our analysis, the $d^{1 / 3}$ is term is consistent with the analytic value 2.1247 in Eq. (15). The sub-leading term $\log _{2} d$ in this expression is very difficult to obtain analytically and we have not succeeded in this task. However, it is clear that there there is an improvement from the value $\frac{1}{6}=0.1666 \ldots$ appearing in (15). The improvement is also evident from the numbers in Table $\mathbb{I}$.

It is worth noting that for large $d$ the optimum gap $\sigma \simeq \frac{2.77 \ldots}{d}$ (from a best fit analysis). This scaling with $d$ is slightly different from that found in the previous section and in [7] (there $\sigma \simeq 1.81 / d$ ). Again notice that the scaling of $\sigma$ with $d, \sigma \propto 1 / d$ is necessary in order not to introduce an exponential suppression of density. In fact for large $d,(1+c / d)^{d} \rightarrow e^{c}$ multiplies the density $\phi$ in all the formulas (and hence it reduces the terminal value by $e^{-c}$ ). A larger gap, say $\mathcal{O}\left(d^{-(1-\epsilon)}\right)$, would suppress the density by an exponentially large amount $e^{-d^{\epsilon}}$.

Table I compares the final results of our analysis for the conjectured lower bound on the maximal density to the previous lower bound, the best known packings, and the optimal upper bound in [4] for selected dimensions up to $d=150$. As in the previous cases, the Yamada condition [29] is violated only for $d=1$. This supports the conclusion reached in 
Ref. [7] that the Yamada condition appears to only have practical relevance in very low dimensions.

TABLE I: Estimates of the maximal densities for selected dimensions up to $d=150 . \phi_{b . k}$ is the densest known packing, $\phi_{C E}$ is the upper bound of Cohn and Elkies, $\phi_{1, *}$ is the terminal density for a single delta function and $\phi_{2, *}$ for two delta functions.

\begin{tabular}{|c|c|c|c|c|}
\hline$d$ & $\phi_{b . k .}$ & $\phi_{C E}$ & $\phi_{*, 1}$ & $\phi_{*, 2}$ \\
\hline 3 & 0.74049 & 0.77982 & 0.57665 & 0.63306 \\
\hline 4 & 0.61685 & 0.64774 & 0.42526 & 0.47885 \\
\hline 5 & 0.46527 & 0.52506 & 0.30591 & 0.35437 \\
\hline 6 & 0.37295 & 0.41776 & 0.21360 & 0.24966 \\
\hline 7 & 0.29530 & 0.32757 & 0.14713 & 0.17991 \\
\hline 8 & 0.25367 & 0.25367 & 0.09985 & 0.12467 \\
\hline 12 & 0.04945 & 0.08384 & 0.01915 & 0.025721 \\
\hline 15 & 0.01685 & 0.03433 & 0.00516 & 0.00722 \\
\hline 19 & 0.004121 & 0.009885 & 0.000845 & 0.001233 \\
\hline 24 & 0.00193 & 0.00193 & $8.24 \times 10^{-5}$ & 0.000125 \\
\hline 31 & $1.18 \times 10^{-5}$ & $1.93 \times 10^{-4}$ & $2.91 \times 10^{-6}$ & $4.57 \times 10^{-6}$ \\
\hline 36 & $6.14 \times 10^{-7}$ & $3.59 \times 10^{-5}$ & $2.57 \times 10^{-7}$ & $4.13 \times 10^{-7}$ \\
\hline 56 & $2.33 \times 10^{-11}$ & - & $1.25 \times 10^{-11}$ & $2.13 \times 10^{-11}$ \\
\hline 60 & $2.97 \times 10^{-13}$ & - & $1.67 \times 10^{-12}$ & $2.87 \times 10^{-12}$ \\
\hline 64 & $1.33 \times 10^{-13}$ & - & $2.22 \times 10^{-13}$ & $3.83 \times 10^{-13}$ \\
\hline 80 & $1.12 \times 10^{-16}$ & - & $6.52 \times 10^{-17}$ & $1.15 \times 10^{-16}$ \\
\hline 100 & - & - & $2.28 \times 10^{-21}$ & $4.11 \times 10^{-21}$ \\
\hline 150 & $8.44 \times 10^{-39}$ & - & $1.27 \times 10^{-32}$ & $2.30 \times 10^{-32}$ \\
\hline
\end{tabular}

\section{CONCLUSIONS AND OPEN QUESTIONS}

The problem of finding the asymptotic behavior of the maximal density $\phi_{\max }$ of sphere packings in high dimensions is one of the most fascinating and challenging problems in geometry. In this paper, we have shown how, using linear programming bounds and a 


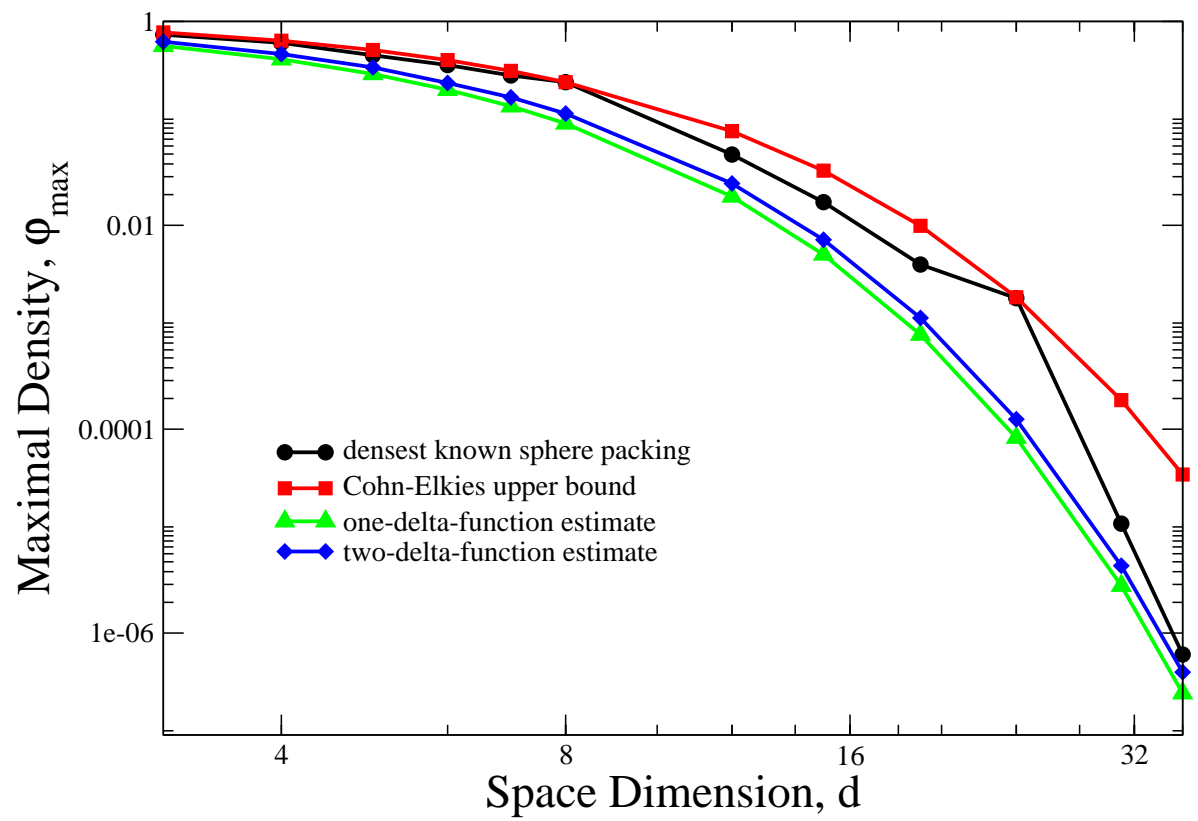

FIG. 4: (Color online) Comparison of different results for the maximal density $\phi_{\max }$ versus dimension $d$. From bottom to top: Torquato-Stillinger result [7] (1-delta function with gap), one of the results of this paper (2-delta functions with a gap), densest known packings [12], and the Cohn-Elkies upper bound [4].

TABLE II: Terminal density $\phi_{*}$ for two delta functions and a gap, corresponding optimal gap $\sigma$, and optimal average kissing number $Z_{1, *}$ for large $d$.

\begin{tabular}{c|c|c|c}
$d$ & $\sigma$ & $Z_{1, *}$ & $\phi_{*}$ \\
\hline 200 & 0.013508 & $1.57 \times 10^{18}$ & $1.06 \times 10^{-43}$ \\
250 & 0.010895 & $7.15 \times 10^{21}$ & $4.18 \times 10^{-55}$ \\
300 & 0.009132 & $2.94 \times 10^{25}$ & $1.49 \times 10^{-66}$ \\
350 & 0.007862 & $1.12 \times 10^{29}$ & $4.96 \times 10^{-78}$ \\
400 & 0.006903 & $2.93 \times 10^{25}$ & $1.56 \times 10^{-89}$ \\
450 & 0.006154 & $1.38 \times 10^{36}$ & $4.73 \times 10^{-101}$ \\
500 & 0.005553 & $4.67 \times 10^{39}$ & $1.40 \times 10^{-112}$
\end{tabular}

conjecture concerning the existence of disordered sphere packings based on pair-correlation information, the asymptotic conjectural lower bound [7]

$$
\phi_{\max } \geq 2^{-(0.77865 \ldots) d},
$$


which provides the putative exponential improvement on Minkowski's century-old lower bound (21), is actually much more general than one could have initially surmised. Precisely the same exponential improvement arises for a simpler pair-correlation function than the one employed in [7] and survives also to a considerable enlargement of the family of test functions $g_{2}$. This family of functions includes two delta functions with a gap (which we have shown improves upon the prefactor multiplying $2^{-(0.77865 \ldots) d}$ given in Ref. [7]) and, we argue, any finite number of delta functions. If this is true, as we believe, it signifies that the decorrelation principle alone has a huge predictive power, since an exponential improvement of Minkowski's bound has proved to be an extremely difficult problem.

One outstanding open question is certainly in which sense this is to be interpreted as an asymptotic bound. Based on our present, limited knowledge of optimal sphere packings, we foresee diverse scenarios. In one case, for sufficiently large $d$, the importance of higherorder correlations is to be neglected altogether and the bound becomes exact by virtue of the decorrelation principle. This would mean that the asymptotic Kabatiansky-Levenshtein upper bound is far from optimal: a provocative possibility. In a second scenario, it could be that "special dimensions" continue to exist for which the negligence of higher-order correlations is impossible. In this case, the lower bound obtained by our methods would not apply to these special dimensions but will continue to apply to the other dimensions. On the other hand, if the frequency of appearance of these dimensions over the integers is decreasing then the decorrelation principle is safe. A third but more pessimistic possibility is that these dimensions are actually becoming more and more frequent, and our conjectural bound would apply only to the subset of dimensions remaining. However, there is absolutely no evidence at present for either the second or third scenario. Our best guess at the moment is that the optimal packings in very high dimensions will possess no symmetry at all and therefore are truly disordered. If so, then the decorrelation principle dictates that pair correlations alone completely characterize the packing in high $d$, implying that the form of the asymptotic bound (42) is exact!

The fact that pair correlations can completely specify an optimal packing may seem to be counterintuitive at first glance, but we can now identify even low dimensions where this phenomenon occurs. Specifically, whenever the linear programming bounds are exact (i.e., achieve some packing), pair correlation information is sufficient to determine the optimal packing! This outcome, in all likelihood, occurs in $\mathbb{R}^{2}, \mathbb{R}^{8}$ and $\mathbb{R}^{24}[4$, 11]. This implies 
that whenever linear programming bounds are not sharp in low dimensions (albeit without a duality gap for any $d$ [33]), information about high-order correlations are required to get optimal solutions.

Another interesting question arises because our procedure, like Minkowski's, is nonconstructive. Specifically, it is an open question whether there exist packing constructions that realize our test $g_{2}$ 's. For future investigations, it would be fruitful to determine whether there are periodic or truly disordered packings that have pair correlation functions that approximate well the ones studied in this paper. If these packings could be identified, one should attempt to ascertain whether the higher-order correlations diminish in importance as $d \rightarrow \infty$ in accordance with the decorrelation principle. If such packings exist (or better, if a $d$-dependent family of them does), they would enable one to place on firm, solid ground the putative exponential improvement on Minkowski's bound. We are currently investigating these questions.

\section{Acknowledgments}

We thank Henry Cohn and Abhinav Kumar for discussions and for making us aware of their unpublished proof that there is no duality gap in the linear programming bounds. This work was supported by the Division of Mathematical Sciences at the National Science Foundation under Grant No. DMS-0312067.

[1] C. E. Shannon, Bell Syst. Tech. J. 27379 (1948); ibid. 623 (1948).

[2] S. Torquato and F. H. Stillinger, J. Phys. Chem. B 106, 8354 (2002); Erratum 106, 11406 (2002).

[3] H. Cohn, Geom. Topol. 6, 329 (2002).

[4] H. Cohn and N. Elkies, Annals Math. 157, 689 (2003).

[5] T. C. Hales, Ann. Math. 162, 1065 (2005).

[6] S. Torquato and F. H. Stillinger, Phys. Rev. E 73, 031106 (2006).

[7] S. Torquato and F. H. Stillinger, Experimental Math. 15, 307 (2006).

[8] G. Parisi and F. Zamponi, J. Stat. Mech. P03017 (2006). 
[9] M. Skoge, A. Donev, F. H. Stillinger and S. Torquato, Phys. Rev. E 74, 041127 (2006).

[10] S. Torquato, O. U. Uche and F. H. Stillinger, Phys. Rev. E 74, 061308 (2006).

[11] H. Cohn and A. Kumar, to be published. See also arXiv:math.MG/0403263.

[12] J. H. Conway and N. J. A. Sloane, Sphere Packings, Lattices and Groups (Springer-Verlag, New York, 1998).

[13] C. A. Rogers, Proc. Lond. Math. Soc. 8, 609, (1958).

[14] C. A. Rogers, Packing and Covering (Cambridge University Press, Cambridge, 1964).

[15] H. L. Frisch and J. K. Percus, Phys. Rev. E 60, 2942 (1999).

[16] G. Parisi and F. Slanina, Phys. Rev. E 62, 6554 (2000).

[17] S. Torquato, Random Heterogeneous Materials: Microstructure and Macroscopic Properties (Springer-Verlag, New York, 2002).

[18] In Ref. [7], a disordered packing in $\mathbb{R}^{d}$ is defined to be a packing in which the pair correlation function $g_{2}(\mathbf{r})$ decays to its long-range value of unity faster than $|\mathbf{r}|^{-d-\epsilon}$ for some $\epsilon>0$.

[19] Already in $\mathbb{R}^{10}$, we encounter for the first time a non-lattice (i.e., periodic) packing that is denser than all known lattice packings; see Ref. [12].

[20] H. Minkowski, J. reine angew. Math. 129, 220 (1905).

[21] K. Ball, Int. Math. Res. Notices 68217 (1992).

[22] See Ref. [7] and references therein.

[23] G. A. Kabatiansky and V. I. Levenshtein, Problems of Information Transmission 14, 1 (1978).

[24] The ghost RSA process is a time-dependent packing in $\mathbb{R}^{d}$ in which there are no spheres at time $t=0$. For any $t>0$, sphere placements are attempted by a purely random process and are accepted if they do not overlap any existing sphere in the packing or any previously rejected sphere at any previous time. Such spheres are called "ghost' spheres. In the infinitetime limit, the ghost RSA packings achieves a maximal density of $2^{-d}$. See Ref. [6] for more details of this packing and its generalizations.

[25] The well-known standard RSA packing is also a time-dependent packing in $\mathbb{R}^{d}$ in which there are no spheres at time $t=0$. For any $t>0$, sphere placements are attempted by a purely random process and are accepted if they do not overlap any current sphere in the packing. In the infinite-time limit, the packing is saturated and its maximal density is achieved.

[26] Based upon our knowledge of spin systems in high dimensions, it may be tempting to conclude that the decorrelation principle is an expected "mean-field" behavior, but this cannot be the 
case for reasons that have already been discussed elsewhere [9, 10].

[27] S. Torquato and F. H. Stillinger, Phys. Rev. E, 68, 041113 (2003); 68, 069901 (2003).

[28] O. Costin and J. Lebowitz. J. Phys. Chem. B. 108, 19614 (2004).

[29] M. Yamada, Prog. Theor. Phys. 25, 579 (1961). The Yamada condition is an inequality involving the variance $\sigma^{2}(\Omega) \equiv\left\langle\left(N(\Omega)^{2}-\langle N(\Omega)\rangle\right)^{2}\right\rangle$ in the number $N(\Omega)$ of particle centers contained within a region or "window" $\Omega \subset \mathbb{R}^{d}$, namely, $\sigma^{2}(\Omega) \geq \theta(1-\theta)$, where $\theta$ is the fractional part of the expected number of points $\rho|\Omega|$ contained in the window. Reference [27] gives an explicit representation of the number variance in terms of the pair correlation function.

[30] J. R. Crawford, S. Torquato, and F. H. Stillinger, J. Chem. Phys. 119, 7065 (2003).

[31] O. U. Uche, F. H. Stillinger and S. Torquato, Physica A 360, 21 (2006).

[32] D. Ruelle, Statistical Mechanics: Rigorous Results (World Scientific, New York, 1999).

[33] H. Cohn and A. Kumar, private communication. 